\title{
Regional Stability of Discrete-Time Linear Systems Subject to Asymmetric Input Saturation*
}

\author{
Leonardo B. Groff
}

João M. Gomes da Silva Jr

\author{
Giorgio Valmorbida
}

\begin{abstract}
This paper studies the problem of estimating the region of attraction of linear discrete-time systems with asymmetric input saturation. Using a Piecewise Quadratic Lyapunov function, we propose conditions for the local stability of the equilibrium. The asymmetric bounds of the saturation are considered in these conditions. Estimates of the region of attraction, possibly asymmetric with respect to the origin, are computed by solving convex optimization problems. Numerical results illustrate the effectiveness of the proposed method.
\end{abstract}

\section{INTRODUCTION}

Actuator saturation appears in every practical control systems due to physical device limits or security reasons. The saturation can impact on the closed-loop system stability and performance. Indeed, even for linear systems subject to input saturation we may face loss of global stability, multiple equilibria, limit cycles, performance degradation and windup of controller states. For the above reasons, actuator saturation has been intensively studied in the last decades and successful and constructive methods for analysis and design have been obtained and are reported for instance in [1], [2] and [3].

It is well known that the global (or semi-global) stabilization of the origin of linear systems with input saturation is possible only if the system is null-controllable [4]. If the plant is exponentially unstable, only the local stability of the origin can be obtained. In this case, since the analytical characterization of the region of attraction of the origin is in general difficult, a method to estimate such a set is to obtain approximations as level sets of local Lyapunov functions. These domains can therefore be considered as "safe" regions of operation or regions of "guaranteed" stability. In this context, approaches considering polyhedral [5], quadratic

\footnotetext{
*This work was financed in part by the Coordenação de Aperfeiçoamento de Pessoal de Nível Superior (CAPES) - Finance Code 001 (STIC AmSud project 88887.143274/2017-00), and CNPQ (Grants PQ - 305979/2015-9 and Univ. 422992/2016-0), Brazil.

L. B. Groff is with the Graduate Program in Electrical Engineering (PPGEE), Universidade Federal do Rio Grande do Sul (UFRGS), av. Osvaldo Aranha, 103, 90035-190 Porto Alegre-RS, Brazil and with the Laboratoire des Signaux et Systèmes, CentraleSupélec, CNRS, Univ. ParisSud, Université Paris-Saclay, 3 Rue Joliot-Curie, 91192 Gif sur Yvette, France. e-mail: leonardo.groffeufrgs.br

J. M. Gomes da Silva Jr. is with the Department of Automation and Energy (DELAE) and the Graduate Program in Electrical Engineering (PPGEE), Universidade Federal do Rio Grande do Sul (UFRGS), av. Osvaldo Aranha, 103, 90035-190 Porto Alegre-RS, Brazil. e-mail: jmgomes@ufrgs.br

G. Valmorbida is with the Laboratoire des Signaux et Systèmes, CentraleSupélec, CNRS, Univ. Paris-Sud, Université Paris-Saclay, 3 Rue Joliot-Curie, 91192 Gif sur Yvette, France. $\mathrm{He}$ is also with Projet DISCO, INRIA Saclay. e-mail: giorgio.valmorbida@l2s. centralesupelec.fr
}

(see [3] and references therein), composite quadratic [6] and piecewise quadratic functions [7], [8] have been proposed in the literature. Furthermore, domains generated by rational and polynomial Lyapunov functions have been considered for nonlinear plants in [9] and [10], for instance.

Note that the above mentioned approaches for computing estimates of the region of attraction assume the input saturation is a symmetric nonlinearity. In many cases, however, as for instance in constant references tracking, the equilibrium point can be translated to the origin and the saturation becomes asymmetric for stability analysis purposes. One possible solution is to consider a worst case scenario with a symmetric saturation bounded by the minimum bound of the asymmetric saturation. This solution is clearly conservative.

On the other hand, asymmetric actuator constraints were considered in early works dealing with the problem of synthesis of state feedback control laws leading to the positive invariance of the region of linear behavior of the closed-loop (see for instance [11] and [12]).In [13], based on the transformation of asymmetric saturation bounds in symmetric ones by means of a bias term added in the control law, a method for computing quadratic domains has been proposed. In [14], a method for computing asymmetric regions of stability for continuous-time systems is presented. It considers a partition of the state space, as proposed in [15], and piecewise quadratic functions obtained from deadzone nonlinearities, as proposed in [8].

In this work we aim at providing estimates of the region of attraction for discrete-time linear systems under asymmetric actuator saturation. Similar to [15] and [14], we consider a partition of the state space in regions obtained by the intersection of half spaces defined from the considered control law. In each of these regions, the closed-loop is described by a symmetric deadzone nonlinearity. Generalized sector conditions for this nonlinearity, as proposed in [16], are used to provide conditions allowing to compute a piecewise quadratic (PWQ) function leading to an asymmetric estimate of the region of attraction. The PWQ Lyapunov function is composed by quadratic functions, defined from different positive definite matrices in each region. Conditions to ensure that such function is decreasing, and thus the origin is stable, are presented, and convex optimization problems are proposed to find an optimized estimate of the region of attraction. Moreover, in order to further reduce conservatism and enhance the obtained estimates, conditions relaxing the need of definite positiveness of the matrices defining the quadratic function in each region are also formulated. Numerical examples illustrate the method and shows the 
considerable conservatism reduction when compared to the worst case symmetric solution above mentioned.

The paper is organized as follows. In Section II, the problem of estimating the region of attraction of asymmetrically saturated systems is stated. In Section III, a piecewise representation based on symmetric deadzone functions is presented. In Section IV, the conditions for the stability of systems subject to asymmetrical input saturation are presented in the form of LMIs, and then a relaxation on the stability conditions is proposed. In Section V convex optimization problems are used to find suitable solutions to these stability conditions. Numerical examples are given in Section VI showing the benefits of the proposed technique, and, finally, some concluding remarks are made in Section VII.

Notation: For $M \in \mathbb{R}^{m \times m}, M^{\top}$ denotes its transpose. $\operatorname{He}(M)=M+M^{\top}$. We use $M>N$ to denote that $M-$ $N$ is positive definite. $I$ and 0 are an identity matrix and a null matrix of appropriate dimensions. $M \succcurlyeq 0$ denotes that the elements in $M$ are non-negative. $\operatorname{diag}\left(M_{1}, \ldots, M_{n}\right)$ denotes the block diagonal matrix whose diagonal blocks are the matrices $M_{1}$ through $M_{n} . M_{(i)}$ denotes the $i$ th row of $M$ and, for $v \in \mathbb{R}^{m}, v_{(i)}$ denotes $i$ th component of $v$. For a function $f: X \rightarrow Y, f[D]$ denotes the image of $D \subseteq$ $X$ under $f$. The decentralized saturation map $\operatorname{sat}(v, \underline{\mu}, \bar{\mu})$ : $\mathbb{R}^{m} \rightarrow \mathbb{R}^{m}$ is defined as

$$
\operatorname{sat}(v, \underline{\mu}, \bar{\mu})_{(l)}:= \begin{cases}-\underline{\mu}_{(l)} & \text { if } v_{(l)}<-\underline{\mu}_{(l)} \\ v_{(l)} & \text { if }-\underline{\mu}_{(l)} \leq v_{(l)} \leq \bar{\mu}_{(l)} \\ \bar{\mu}_{(l)} & \text { if } v_{(l)}>\bar{\mu}_{(l)}\end{cases}
$$

with $\underline{\mu}_{(l)}>0$ and $\bar{\mu}_{(l)}>0, \forall l=1,2, \ldots, m$. For the symmetric saturation case, i.e. when $\bar{\mu}=\mu=\mu$, we use a shortcut notation

$$
\operatorname{sat}(v, \mu)=\operatorname{sat}(v, \mu, \mu) .
$$

The deadzone map $\phi(v, \underline{\mu}, \bar{\mu}): \mathbb{R}^{m} \rightarrow \mathbb{R}^{m}$ is defined as

$$
\phi(v, \underline{\mu}, \bar{\mu}):=v-\operatorname{sat}(v, \underline{\mu}, \bar{\mu}) .
$$

Similarly, we adopt the shortcut notation $\phi(v, \mu)=$ $\phi(v, \mu, \mu)$.

\section{Problem Statement}

Consider the discrete-time system

$$
x(k+1)=\bar{A} x(k)+B \operatorname{sat}(K x(k), \underline{\mu}, \bar{\mu})
$$

where $x \in \mathbb{R}^{n}$ is the state vector, and $\operatorname{sat}(K x, \mu, \bar{\mu})$ is the saturation as defined in (1), $\bar{A} \in \mathbb{R}^{n \times n}, B \in \mathbb{R}^{n \times m}, K \in$ $\mathbb{R}^{m \times n}$ are real constant matrices.

Using (3) the system (4) can be rewritten as

$$
x(k+1)=A x(k)-B \phi(K x(k), \underline{\mu}, \bar{\mu}),
$$

with $A \in \mathbb{R}^{n \times n}=\bar{A}+B K$.

We suppose that $K$ has been computed such that $A$ is a Schur-Cohn matrix, i.e. the origin of system (5) (or equivalently (4)) is asymptotically stable. However, due to the presence of the nonlinearity, the global stability of the origin is, in general, not guaranteed [4]. Indeed, this is always the case when the open-loop system is not exponentially stable, i.e. when $\bar{A}$ is not Schur-Cohn stable. In this case, to characterize the set of initial conditions from which the trajectories converge to the origin of $(5)$, let $\zeta\left(k, x_{0}\right)$ be the solution of (5) starting at $x_{0}, k \in \mathbb{N}$. The following definition of the region of attraction (RA) of the origin can be stated.

Definition 1: The RA of the origin of a system is the set of all initial conditions $x_{0}$ such that $\zeta\left(k, x_{0}\right) \rightarrow 0$ when $k \rightarrow \infty$.

The exact characterization of the RA is generally a complex task [17]. We thus define a region of asymptotic convergence (RAC) as follows.

Definition 2: A region $\mathcal{R}$ is a $\mathrm{RAC}$ with respect to the origin of a system if $0 \in \mathcal{R}$ and $\zeta\left(k, x_{0}\right) \rightarrow 0$ when $k \rightarrow \infty$, $\forall x_{0} \in \mathcal{R}$.

A RAC is a region of guaranteed convergent behavior and can be used as an estimate of the true RA of the origin. Note that a RAC will be always included in the RA. Thus, the problem we are interested in regards the determination of a RAC for system (5), as large as possible with respect to some size criterion, taking explicitly into account that the saturation limits are asymmetric. Differently from previous approaches in the literature, we focus on the determination of asymmetric regions to best cope with the asymmetric constraints.

\section{Piecewise Representation of the System}

Similarly to [14], [15], we consider a partition of the state space in regions defined by the hyperplanes $K_{(l)} x=0, l=$ $1, \ldots, m$.

With this aim, define the diagonal matrix

$$
\Lambda_{i}:=\operatorname{diag}\left(\lambda_{i, 1}, \ldots, \lambda_{i, m}\right)
$$

with $\lambda_{i, j} \in\{-1,1\}, j=1, \ldots, m, i=1, \ldots, m$. Thus, there are $2^{m}$ matrices $\Lambda_{i}$. Associated to each matrix $\Lambda_{i}$ we can therefore define the following set:

$$
\Gamma_{i}:=\left\{x \in \mathbb{R}^{n} \mid \Lambda_{i} K x \succcurlyeq 0\right\},
$$

Note that each $\Gamma_{i}$ corresponds to a given intersection of half-spaces $\pm K_{(l)} x \geq 0$. Suppose now that $x$ belongs to a region $\Gamma_{i}$ such that, for instance, $K_{(l)} x \geq 0$. Inside this region it follows that $\phi_{(l)}(K x(k), \underline{\mu}, \bar{\mu})=\phi_{(l)}(K x(k), \bar{\mu})$. On the other hand, if $-K_{(l)} x \geq 0$, then $\phi_{(l)}(K x(k), \underline{\mu}, \bar{\mu})=$ $\phi_{(l)}(K x(k), \underline{\mu})$. Thus, if $x \in \Gamma_{i}$, it follows that

$$
\phi_{(l)}(K x, \underline{\mu}, \bar{\mu})=\phi_{(l)}\left(K x, \mu_{i(l)}\right)
$$

with the elements of the vector $\mu_{i}$ given by

$$
\mu_{i(l)}=\left\{\begin{array}{l}
\mu_{(l)} \text { if } \lambda_{i, l}=-1 \\
\bar{\mu}_{(l)} \text { if } \lambda_{i, l}=1
\end{array} .\right.
$$

For shortness of notation, let us define

$$
\phi_{i}(K x):=\phi\left(K x, \mu_{i}\right) .
$$

It follows that the system (5) is equivalently given by the piecewise model

$$
x(k+1)=A x(k)-B \phi_{i}(K x(k)), \text { if } x(k) \in \Gamma_{i} .
$$


Also, for system (6) define the set

$$
\Omega:=\left\{(i, j) \in \mathbb{N}^{2} \mid x(k) \in \Gamma_{i}, x(k+1) \in \Gamma_{j}\right\}
$$

that represents the possible transitions between sets $\Gamma_{i}$. Such a set can be determined by reachability analysis [18]. In short, considering $x(k+1)=f(x)$, the possible transitions from $\Gamma_{i}$ are defined by the sets $\Gamma_{j}$ which intersect $f\left[\Gamma_{i}\right]$.

\section{Main Results}

In this section we present conditions for the local stability of system (6). For such, the following PWQ Lyapunov function candidate is considered

$$
V(x)=V_{i}(x) \text { if } x \in \Gamma_{i}, \forall i=1, \ldots, 2^{m},
$$

with

$$
V_{i}(x)=x^{\top} P_{i} x
$$

Theorem 1: If there exist matrices $W_{i} \in \mathbb{R}^{n \times n}=W_{i}^{\top}>$ $0, Y_{i} \in \mathbb{R}^{m \times n}$ and diagonal matrices $U_{i, j} \in \mathbb{R}^{m \times m}>0$, $i, j \in\left\{1, \ldots, 2^{m}\right\}$, such that

$$
\begin{gathered}
\operatorname{He}\left(\left[\begin{array}{ccc}
-\frac{1}{2} W_{i} & Y_{i}^{\top} & W_{i} A^{\top} \\
0 & -U_{i, j} & -U_{i, j} B^{\top} \\
0 & 0 & -\frac{1}{2} W_{j}
\end{array}\right]\right)<0, \forall(i, j) \in \Omega \\
\operatorname{He}\left(\left[\begin{array}{cc}
\frac{1}{2} W_{i} & W_{i} K_{(l)}^{\top}-Y_{i(l)}^{\top} \\
0 & \frac{1}{2} \mu_{i(l)}^{2}
\end{array}\right]\right) \geq 0, \begin{array}{l}
\forall i=1, \ldots, 2^{m}, \\
\forall l=1, \ldots, m,
\end{array}
\end{gathered}
$$

hold, then the origin of system (6) is asymptotically stable. Furthermore, the region

$$
\mathcal{R}=\tilde{\mathcal{R}}_{1} \cup \ldots \cup \tilde{\mathcal{R}}_{2^{m}},
$$

where $\tilde{\mathcal{R}}_{i}=\mathcal{E}\left(W_{i}^{-1}\right) \cap \Gamma_{i}$, with $\mathcal{E}\left(W_{i}^{-1}\right)=\{x \in$ $\left.\mathbb{R}^{n} \mid x^{\top} W_{i}^{-1} x \leq 1\right\}$, is a RAC.

Proof: Suppose that $x(k) \in \Gamma_{i}$ and consider $(i, j) \in \Omega$. Then, we have

$$
\Delta V(x(k))=x(k+1)^{\top} P_{j} x(k+1)-x(k)^{\top} P_{i} x(k)
$$

for some $j$ such that $(i, j) \in \Omega$. Thus, if

$$
x(k+1)^{\top} P_{j} x(k+1)-x(k)^{\top} P_{i} x(k)<0, \forall(i, j) \in \Omega
$$

holds, then $\Delta V(x(k))<0 \forall x \in \mathbb{R}^{n}$. Using (6), we must therefore ensure that

$$
\xi_{i}(k)^{\top} \Pi^{\top} P_{j} \Pi \xi_{i}(k)-x(k)^{\top} P_{i} x(k)<0, \forall(i, j) \in \Omega .
$$

with

$$
\Pi=\left[\begin{array}{ll}
A & -B
\end{array}\right]
$$

and

$$
\xi_{i}(k)=\left[x(k)^{\top} \quad \phi_{i}(K x(k))^{\top}\right]^{\top} .
$$

Now, for $G_{i} \in \mathbb{R}^{m \times n}, i=1, \ldots, m$, consider the sets

$$
\mathcal{S}_{i}:=\left\{x \in \mathbb{R}^{n}\left|\mu_{i}-\right|\left(K-G_{i}\right) x \mid \succcurlyeq 0\right\} .
$$

According to the Lemma 1 in [16], if $x \in \mathcal{S}_{i}$, then

$$
2 \phi_{i}(K x)^{\top} T_{i, j}\left(\phi_{i}(K x)-G_{i} x\right) \leq 0,
$$

that is

$$
\xi_{i}(k)^{\top}\left(\operatorname{He}\left(\left[\begin{array}{cc}
0 & G_{i}^{\top} T_{i, j} \\
0 & -T_{i, j}
\end{array}\right]\right)\right) \xi_{i}(k) \geq 0
$$

holds for any positive diagonal matrix $T_{i, j}$. Thus, from (12) and (13), if

$$
\begin{array}{r}
\xi_{i}(k)^{\top}\left(\operatorname{He}\left(\left[\begin{array}{cc}
-\frac{1}{2} P_{i} & G_{i}^{\top} T_{i, j} \\
0 & -T_{i, j}
\end{array}\right]\right)+\Pi^{\top} P_{j} \Pi\right) \xi_{i}(k)<0, \\
\forall(i, j) \in \Omega . \quad(14)
\end{array}
$$

holds, then $\Delta V<0$ provided that $x \in \mathcal{S}_{i}$.

From Schur's complement, we have that the above quadratic form is negative definite if and only if

$$
\mathrm{He}\left(\left[\begin{array}{ccc}
-\frac{1}{2} P_{i} & G_{i}{ }^{\top} T_{i, j} & A^{\top} \\
0 & -T_{i, j} & -B^{\top} \\
0 & 0 & -\frac{1}{2} P_{j}^{-1}
\end{array}\right]\right)<0, \forall(i, j) \in \Omega .
$$

Applying a congruence transformation defined by $\operatorname{diag}\left(W_{i}, U_{i, j}, I\right)$, with $W_{i}=P_{i}^{-1}, U_{i, j}=T_{i, j}{ }^{-1}, \forall i, j=$ $1, \ldots, 2^{m}$, and defining $Y_{i}=G_{i} W_{i}$, we obtain (7).

Now, we show that $x(k) \in \mathcal{S}_{i}, \forall k \geq 0$ for some $i \in$ $\left\{1, \ldots, 2^{m}\right\}$, provided that $x(0) \in \mathcal{R}$, with $\mathcal{R}$ defined in (9). Note that if

$$
\begin{array}{r}
x(k)^{\top}\left(K_{(l)}-G_{i(l)}\right)^{\top}\left(K_{(l)}-G_{i(l)}\right) \mu_{i(l)}^{-2} x(k) \leq \\
x(k)^{\top} P_{i} x(k), \quad \forall i=1, \ldots, 2^{m}, \quad l=1, \ldots, m
\end{array}
$$

holds, then $x(k) \in \mathcal{S}_{i}$ whenever $x(k)^{\top} P_{i} x(k) \leq 1$. By Schur's Complement, this is equivalent to

$$
\operatorname{He}\left(\left[\begin{array}{cc}
\frac{1}{2} P_{i} & K_{(l)}^{\top}-G_{i}^{\top} \\
0 & \frac{1}{2} \mu_{i(l)}^{2}
\end{array}\right]\right) \geq 0 . \quad \begin{aligned}
& \forall i=1, \ldots, 2^{m}, \\
& \forall l=1, \ldots, m,
\end{aligned}
$$

Applying a congruence transformation defined by $\operatorname{diag}\left(W_{i}, 1\right)$ to (15) yields (8), so if (8) holds, then $x(k) \in \mathcal{S}_{i}$ whenever $x(k)^{\top} P_{i} x(k) \leq 1$, which means that $x(k) \in \mathcal{S}_{i}$, provided $x(k) \in \mathcal{E}\left(P_{i}\right) \cap \bar{\Gamma}_{i}=\tilde{\mathcal{R}}_{i}$.

Now suppose that $x(k) \in \mathcal{E}\left(P_{i}\right) \cap \Gamma_{i}$ and $x(k+1) \in \Gamma_{i}$. Then $x(k)^{\top} P_{i} x(k) \leq 1$ and $x(k) \in \mathcal{S}_{i}$. Since (7) ensures that $x(k+1)^{\top} P_{i} x(k+1)<x(k)^{\top} P_{i} x(k)$ whenever $x(k) \in \mathcal{S}_{i}$, then we can guarantee that $x(k+1)^{\top} P_{i} x(k+1)<1$ and, from (8), $x(k+1) \in \mathcal{S}_{i}$.

On the other hand, suppose that $x(k) \in \mathcal{E}\left(P_{i}\right) \cap \Gamma_{i}$ and $x(k+1) \in \Gamma_{j}$, with $j \neq i$. Then $x(k)^{\top} P_{i} x(k) \leq 1$ and $x(k) \in \mathcal{S}_{i}$. Since, from (7), we have that $V(x(k+1))=$ $x(k+1)^{\top} P_{j} x(k+1)<x(k)^{\top} P_{i} x(k)=V(x(k))$ is verified whenever $x(k) \in \mathcal{S}_{i}$, it follows that $x(k+1)^{\top} P_{j} x(k+1)<1$ and thus, from (8), $x(k+1) \in \mathcal{S}_{j}$.

Finally, note that if $x(0) \in \mathcal{R}$, then $x(0)$ will belong to some $\tilde{\mathcal{R}}_{i}$ and thus to $\mathcal{S}_{i}$. Then, from the reasoning above it follows that $x(k)$ will belong to some $\tilde{\mathcal{R}}_{j}, j \in\left\{1, \ldots, 2^{m}\right\}$, and thus to $\mathcal{S}_{j}, \forall k \geq 0$. Hence, we conclude that (7) jointly with (8) ensure the asymptotic stability of the origin of the system, and that $\mathcal{R}$ is included in the RA of the origin of system (6), i.e. it is a RAC.

We now propose relaxed conditions based on the results in [19]. These conditions will explicitly account for the definition of the sets $\Gamma_{i}$ when verifying inequalities that must 
hold only in such sets. This will allow to relax the need of definite positiveness for matrices $P_{i}$.

Let us define the matrix

$$
E_{i}=\Lambda_{i} K
$$

and consider the following lemma.

Lemma 1: If there exist symmetric matrices $P_{i}$ and $M_{i}$, with $M_{i} \succcurlyeq 0$, such that the matrix inequalities

$$
P_{i}-E_{i}^{\top} M_{i} E_{i}>0, \forall i=1, \ldots, 2^{m}
$$

are verified, then

$$
x^{\top} P_{i} x>0, \quad \forall x \in \Gamma_{i}, x \neq 0, i=1, \ldots 2^{m}
$$

holds.

Proof: Suppose that $x \in \Gamma_{i}$. In this case $E_{i} x \succcurlyeq 0$, and since $M_{i} \succcurlyeq 0$, it follows that $x E_{i}^{\top} M_{i} E_{i} x>0$, which implies from (16) that $x^{\top} P_{i} x>0$.

Lemma 1 is instrumental for the theorem below.

Theorem 2: If there exist symmetric matrices $P_{i}=P_{i}^{\top} \in$ $\mathbb{R}^{n \times n}, M_{i, j} \succcurlyeq 0 \in \mathbb{R}^{m \times m}, \tilde{M}_{i, j} \succcurlyeq 0 \in \mathbb{R}^{m \times m}$ and $N_{i} \succcurlyeq$ $0 \in \mathbb{R}^{m \times m}$, matrices $G_{i} \in \mathbb{R}^{m \times n}$ and diagonal matrices $T_{i, j} \in \mathbb{R}^{m \times m}, i, j \in\left\{1, \ldots, 2^{m}\right\}$, such that

$$
\begin{gathered}
\operatorname{He}\left(\left[\begin{array}{cc}
-\frac{1}{2} P_{i}+E_{i}^{\top} M_{i, j} E_{i} & G_{i}^{\top} T_{i, j} \\
0 & -T_{i, j}
\end{array}\right]\right) \\
+\Pi^{\top}\left(P_{j}+E_{j}^{\top} \tilde{M}_{i, j} E_{j}\right) \Pi<0 \quad \forall(i, j) \in \Omega, \\
\operatorname{He}\left(\left[\begin{array}{cc}
\frac{1}{2} P_{i}-E_{i}^{\top} N_{i} E_{i} & K_{(l)}^{\top}-G_{i(l)}^{\top} \\
0 & \frac{1}{2} \mu_{i(l)}^{2}
\end{array}\right]\right) \geq 0, \\
\forall i=1, \ldots, 2^{m}, \quad \forall l=1, \ldots, m,
\end{gathered}
$$

with $\Pi=\left[\begin{array}{ll}A & -B\end{array}\right]$, hold, then the origin of system (6) is asymptotically stable. Furthermore, the region

$$
\mathcal{R}=\tilde{\mathcal{R}}_{1} \cup \ldots \cup \tilde{\mathcal{R}}_{2^{m}},
$$

where $\tilde{\mathcal{R}}_{i}=\mathcal{E}\left(P_{i}\right) \cap \Gamma_{i}$, with $\mathcal{E}\left(P_{i}\right)=\left\{x \in \mathbb{R}^{n} \mid x^{\top} P_{i} x \leq\right.$ $1\}$, is a RAC.

Proof: From lemma 1, if (17) is verified, then (14) is also verified. Furthermore, if (18) is verified, then so is (15). The rest of the proof comes from following the same steps done in the proof of Theorem 1.

Remark 1: In Theorem 2 the conditions for the positivity of $V(x)$ do not require $P_{i}$ to be positive definite. Note that although the entries of matrices $M_{i, j}$ and $N_{i}$ in (17)-(18) are non-negative, this does not imply that the matrices are positive semi-definite. Moreover, if (17) holds, then $V_{j}(x(k+1))-V_{i}(x(k))<0$ for $x(k) \in \Gamma_{i}$ and $x(k+1) \in \Gamma_{j}, \forall(i, j) \in \Omega$.

\section{Optimization Problems}

The results in the Section IV allow to check whether the origin of the systems is asymptotically stable and provides an estimate of the RA of the origin of the system. In this section we provide a set of optimization problems aiming to obtain a RAC as large as possible. Therefore, we wish to compute, among all possible solution verifying LMIs (7) and (8), the best RAC given by a region $\mathcal{R}$, with respect to some size criterion. For instance, the following optimization problems can thus be proposed.

\section{A. Volume Maximization}

An intuitive and useful size criterion is to maximize the volume of the ellipsoids $\mathcal{E}\left(P_{i}\right)$, aiming at optimizing the volume of $\tilde{\mathcal{R}}_{i}$, and, by consequence, the volume of $\mathcal{R}$. The volume of an ellipsoid $\mathcal{E}\left(P_{i}\right)$ is proportional to $\sqrt{\operatorname{det}\left(W_{i}\right)}$ [3]. Then, we can optimize the volume of $\mathcal{R}$ through the following optimization problem.

Optimization Problem 1 (OP1):

$$
\begin{array}{ll}
\operatorname{maximize} & \sum_{i=1}^{2^{m}} \alpha_{i} \log \left(\operatorname{det}\left(W_{i}\right)\right) \\
\text { subject to } & (7), \quad(8)
\end{array}
$$

where $\alpha_{i} \geq 0$ are scalars allowing to prioritize the maximization in particular regions $\Gamma_{i}$.

\section{B. Maximization of a Shape Set}

Another criterion is to enlarge the RAC in the directions defined by inner shape sets $\mathcal{E}\left(Q_{i}, \gamma_{i}\right)=\left\{x \in \mathbb{R}^{n} \mid x^{\top} Q_{i} x \leq\right.$ $\left.\gamma_{i}^{-1}\right\}$, with $Q_{i}=Q_{i}^{\top}, \gamma_{i}>0, i=1, \ldots, 2^{n}$. The idea consists in determining a RAC such that $\mathcal{E}\left(P_{i}\right) \supset \mathcal{E}\left(Q_{i}, \gamma_{i}\right)$, with $\gamma_{i}$ minimized. This inclusion is obtained with the constraints

$$
\operatorname{He}\left[\begin{array}{cc}
\frac{1}{2} W_{i} & I \\
0 & \frac{\gamma_{i}}{2} Q_{i}
\end{array}\right] \geq 0, i=1, \ldots, 2^{m} .
$$

which imply that $W_{i} \geq \gamma_{i}^{-1} Q_{i}^{-1}$, and, consequently, $P_{i} \leq$ $\gamma_{i} Q_{i}$, so that $x \in \mathcal{E}\left(P_{i}\right)$ whenever $x \in \mathcal{E}\left(Q_{i}, \gamma_{i}\right)$.

Hence, the following optimization problem can be formulated.

Optimization Problem 2 (OP2):

$$
\begin{array}{ll}
\operatorname{minimize} & \sum_{i=1}^{2^{m}} \alpha_{i} \gamma_{i} \\
\text { subject to } & (7), \quad(8),
\end{array}
$$

where $\alpha_{i} \geq 0$ are scalars allowing to prioritize the maximization of the shape set associated to each region.

A similar optimization problem can be formulated using conditions of Theorem 2, considering the variables $P_{i}$ as follows.

Optimization Problem 3 (OP3):

$$
\begin{aligned}
\operatorname{minimize} & \sum_{i=1}^{2^{m}} \alpha_{i} \gamma_{i} \\
\text { subject to } & (17), \quad(18), \\
& P_{i}+E_{i}^{\top} X_{i} E_{i}-\gamma_{i} Q_{i}<0, \\
& X_{i}=X_{i}^{\top} \succcurlyeq 0 .
\end{aligned}
$$

Note that in this case $x \in \mathcal{E}\left(Q_{i}, \gamma_{i}\right)$ implies $x \in \mathcal{E}\left(P_{i}\right)$ only if $x \in \Gamma_{i}$.

It should be pointed out that even though Theorem 2 is less conservative than Theorem 1, the inequalities (17) are not LMIs due to the product between variables $G_{i}$ and $T_{i, j}$. In this case the optimization problems become non-convex. One possible solution is to first solve the Optimization Problem 2 
to obtain $U_{i, j}$, and then consider $T_{i, j}=U_{i, j}^{-1}$ to solve Optmization Problem 3 and find a sub-optimal solution. Other solution is to apply the following iterative algorithm:

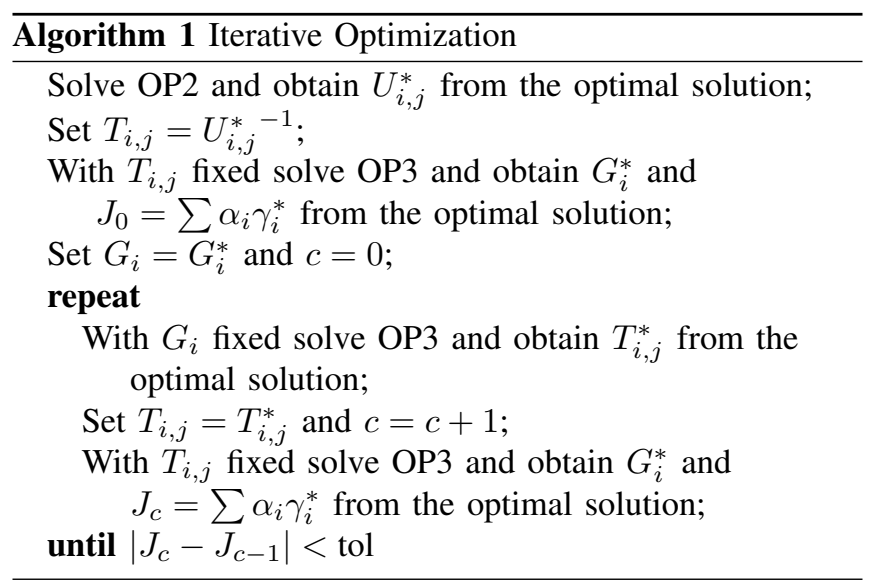

\section{NUMERICAL EXAMPLES}

\section{A. Example 1}

Consider system (5) with

$$
\begin{gathered}
A=\left[\begin{array}{cc}
0.2 & 1 \\
-0.05 & 1
\end{array}\right], \quad B=\left[\begin{array}{l}
1 \\
0
\end{array}\right] \\
K=\left[\begin{array}{ll}
-1 & 1
\end{array}\right],
\end{gathered}
$$

taken from [16], where a symmetric saturation is considered. We consider now asymmetric saturation bounds given by $\underline{\mu}=1, \bar{\mu}=6$. For this system, we have that

$$
\Omega=\{(1,1),(1,2),(2,1),(2,2)\} .
$$

Considering Optimization Problem 1, we wish to calculate a $\operatorname{RAC} \mathcal{R}$, as defined in (9), with a volume as large as possible. To this end, we can choose values for the weighing parameters $\alpha_{1}$ and $\alpha_{2}$ related to the volume of the ellipsoid associated to regions $\Gamma_{1}$ and $\Gamma_{2}$, respectively. Choosing weights $\alpha_{1}=\alpha_{2}=1$ we find:

$P_{1}=\left[\begin{array}{cc}0.0926 & -0.0879 \\ -0.0879 & 0.1662\end{array}\right], \quad P_{2}=\left[\begin{array}{cc}0.0183 & -0.0145 \\ -0.0145 & 0.0937\end{array}\right]$

For comparison purposes, we can apply the stability conditions presented in [3], assuming a worst case symmetric saturation bound, i.e. $\mu_{0}=\min \{\bar{\mu}, \underline{\mu}\}=1$ to find a quadratic RAC given by $\mathcal{E}(P)$ with

$$
P=\left[\begin{array}{cc}
0.0732 & -0.0642 \\
-0.0642 & 0.1533
\end{array}\right] \text {. }
$$

In Figure 1, the asymmetric region of stability $\mathcal{R}$ is depicted in red continuous line, while the domain associated to the symmetric worst case approach (obtained with a quadratic Lyaunov function) is shown in dashed line. It can be observed a considerable improvement on the obtained estimate of the region of attraction considering the proposed method, which considers the effective asymmetry of the saturation bounds.

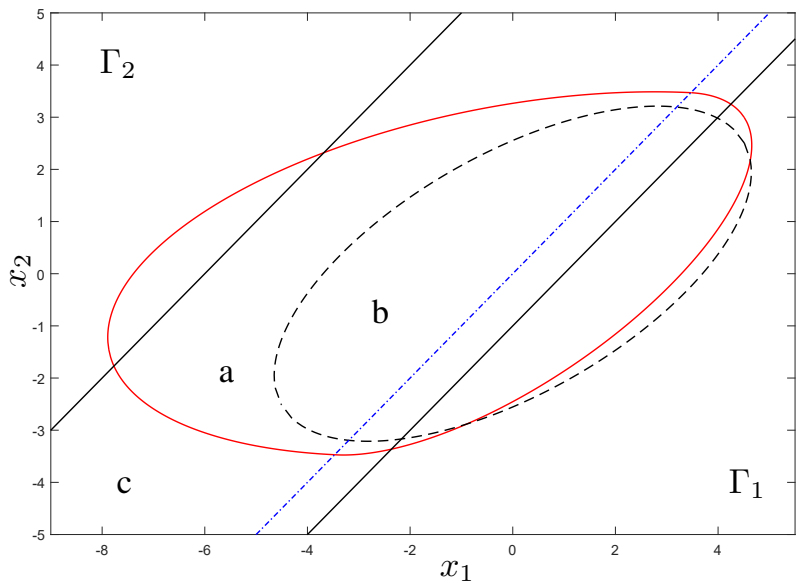

Fig. 1. Example 1 - (a). RAC obtained considering asymmetric bounds (b). RAC obtained considering a symmetric worst case; (c) Region of linear behavior, i.e. $\left\{x \in \mathbb{R}^{2} \mid-1 \leq K x \leq 6\right\}$. The dash-dotted line divides the space in regions $\Gamma_{1}$ and $\Gamma_{2}$.

\section{B. Example 2}

Consider a MIMO system with

$$
\begin{aligned}
& A=\left[\begin{array}{cc}
0.6408 & -0.4663 \\
0.0985 & 0.6620
\end{array}\right], \quad B=\left[\begin{array}{ll}
0.3157 & 0.2623 \\
0.0574 & 0.4052
\end{array}\right] \\
& K=\left[\begin{array}{cc}
-2 & 2 \\
-0.5 & -1.5
\end{array}\right], \underline{\mu}=\left[\begin{array}{ll}
1 & 1
\end{array}\right]^{\top}, \bar{\mu}=\left[\begin{array}{ll}
2 & 2
\end{array}\right]^{\top} \text {. }
\end{aligned}
$$

For this system, we have

$\Omega=\{(1,1),(2,2),(3,3),(4,4),(1,2),(2,4),(4,3),(3,1)\}$.

Defining $Q_{1}=Q_{2}=Q_{3}=Q_{4}=I$, solving the Optimization Problem 2, and subsequently applying Algorithm 1, we find:

$\begin{array}{ll}P_{1}=\left[\begin{array}{cc}0.9651 & -0.5609 \\ -0.5609 & 3.1838\end{array}\right], & P_{2}=\left[\begin{array}{cc}0.8117 & -0.1413 \\ -0.1413 & 1.2259\end{array}\right] \\ P_{3}=\left[\begin{array}{cc}1.1679 & -0.5097 \\ -0.5097 & 2.3593\end{array}\right], & P_{4}=\left[\begin{array}{cc}0.7275 & -0.1745 \\ -0.1745 & 1.4255\end{array}\right]\end{array}$

The obtained RAC $\mathcal{R}$ is presented in red continuous line in Figure 2, as well as some trajectories of initial conditions $x_{0} \in \mathcal{R}$. Figure 3 shows a comparison with the symmetric worst case. As in the previous example, the proposed method lead to a less conservative estimate of the region of attraction than the one obtained from a quadratic function and symmetric worst case saturation bounds. Finally, Figure 4 shows the evolution of the control signal for the initial condition $x(0)=\left[\begin{array}{ll}0.1320 & 0.9121\end{array}\right]^{\top} \in \mathcal{R}$. Note the effective saturation of the control signals on the upper and lower asymmetric bounds.

\section{CONCLUDING REMARKS}

This paper studied the problem of estimating the domain of attraction of linear discrete-time systems under asymmetric input saturation. Sufficient conditions for the asymptotic stability of the origin of the closed-loop systems were derived using a PWQ Lyapunov function. Convex optimization problems were formulated to find an optimized estimate of the region of attraction with respect to some criteria. Less 


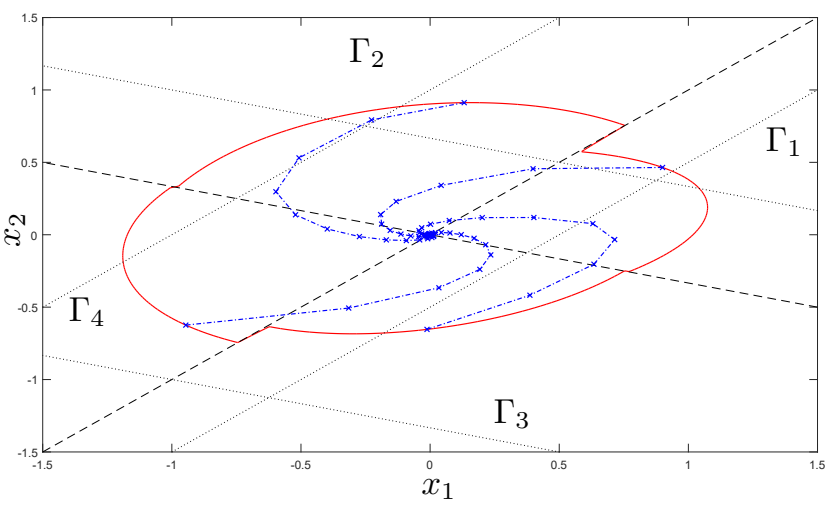

Fig. 2. Example 2 - RAC obtained considering asymmetric bounds; The dashed lines divide the state space in regions $\Gamma_{i}$; The dotted lines depict the saturation limits; In dash-dotted lines, trajectories belonging to the RAC.

conservative conditions are therefore further proposed to relax the need of definite positiveness of the matrices that define the piecewise quadratic function.

Numerical examples illustrate a considerable improvement of the estimates of the region of attraction obtained with the propsosed approach in comparison to the ones obtained with a quadratic Lyapunov function.

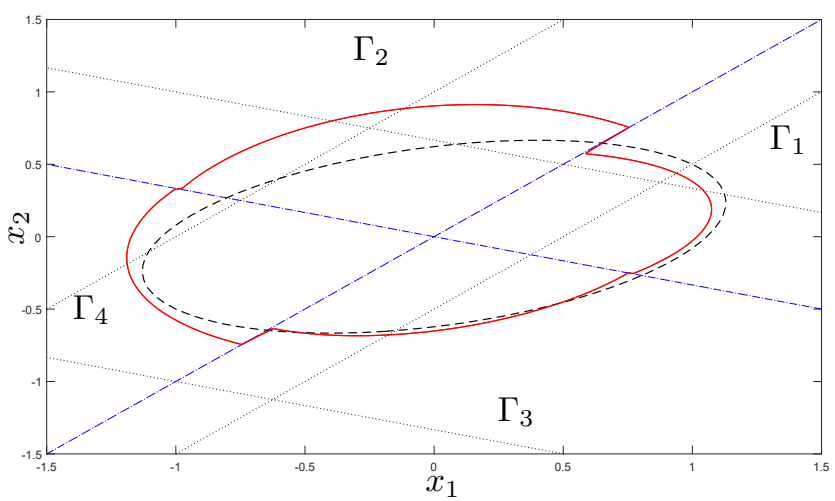

Fig. 3. Example 2 - The solid line depicts $\mathcal{R}$; The dashed line shows the RAC obtained for the symmetric case; The dotted lines depict the saturation limits; In dash-dotted lines, boundaries of the sets $\Gamma_{i}$.

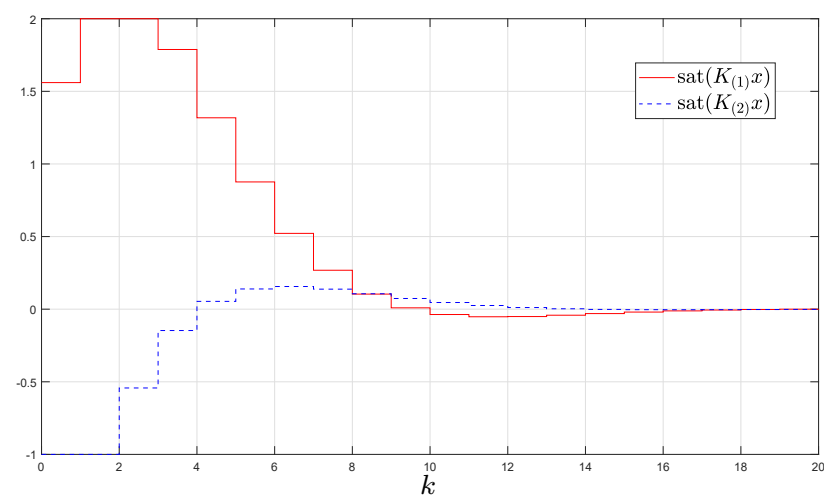

Fig. 4. Evolution of the input signal for $x(0)=\left[\begin{array}{ll}0.1320 & 0.9121\end{array}\right]^{\top}$
We are currently studying strategies to design the control laws. The main difficulty resides in the use of the partition induced by the control law, which is unknown.

\section{REFERENCES}

[1] T. Hu and Z. Lin, Control systems with actuator stauration: Analysis and design. Boston: Birkhauser, 2001.

[2] L. Zaccarian and A. R. Teel, Modern anti-windup synthesis: control augmentation for actuator saturation. Princeton University Press, 2011, vol. 36

[3] S. Tarbouriech, G. Garcia, J. M. Gomes da Silva Jr., and I. Queinnec, Stability and stabilization of linear systems with saturating actuators. London: Springer-Verlag, 2011.

[4] H. J. Sussmann, S. D. Sontag, and Y. Yang, "A general result on the stabilization of linear systems using bounded controls," IEEE Transactions on Automatic Control, vol. 39, no. 12, pp. 2411-2425, 1994.

[5] J. Gomes da Silva Jr. and S. Tarbouriech, "Polyhedral regions of local stability for linear discrete-time systems with saturating controls," IEEE Trans. on Automatic Control, vol. 44, no. 11, pp. 2081-2085, 1999.

[6] T. Hu and Z. Lin, "Composite quadratic Lyapunov functions for constrained control systems," IEEE Transactions on Automatic Control, vol. 48, no. 3, pp. 440-450, Mar 2003.

[7] M. Johansson and A. Rantzer, "Computation of piecewise quadratic Lyapunov functions for hybrid systems," IEEE Transactions on Automatic Control, vol. 43, no. 4, pp. 555-559, 1998.

[8] D. Dai, T. Hu, A. Teel, and L. Zaccarian, "Piecewise-quadratic Lyapunov functions for systems with deadzones or saturations," Systems \& Control Letters Control, vol. 58, no. 5, pp. 365-371, 2009.

[9] D. F. Coutinho and J. M. Gomes da Silva Jr, "Computing estimates of the region of attraction for rational control systems with saturating actuators," IET Control Theory Applications, vol. 4, no. 3, pp. 315325, March 2010.

[10] G. Valmorbida, S. Tarbouriech, and G. Garcia, "Design of polynomial control laws for polynomial systems subject to actuator saturation," IEEE Transactions on Automatic Control, vol. 58, no. 7, pp. 17581770, July 2013.

[11] A. Benzaouia and C. Burgat, "Regulator problem for linear discretetime systems with nonsymmetrical constrained control," Int. Journal of Control, vol. 48, no. 6, pp. 2442-2451, 1988.

[12] E. B. Castelan, J. M. Gomes da Silva Jr., and J. E. R. Cury, "A reduced order framework applied to linear systems with constrained controls," IEEE Trans. on Automatic Control, vol. 41, no. 2, pp. 249-255, 1996.

[13] M. Benhayoun, A. Benzaouia, F. Mesquine, and A. E. Hajjaji, "Stabilization of unsymmetrical saturated discrete-time systems: An LMI approach," in 3rd International Conference on Systems and Control, Algiers, Algeria, Oct 2013, pp. 478-483.

[14] Y. Li and Z. Lin, "An asymmetric Lyapunov function for linear systems with asymmetric actuator saturation," International Journal of Robust and Nonlinear Control, vol. 28, no. 5, pp. 1624-1640, 2018.

[15] C. Yuan and F. Wu, "Switching control of linear systems subject to asymmetric actuator saturation," International Journal of Control, vol. 88, no. 1, pp. 204-215, 2015.

[16] J. M. Gomes da Silva Jr. and S. Tarbouriech, "Anti-windup design with guaranteed region of stability: an LMI-based approach," IEEE Trans. on Automatic Control, vol. 50, no. 1, pp. 106-111, 2005.

[17] H. K. Khalil, Nonlinear Systems, 2nd ed. Upper Saddle River: Prentice-Hall, 1996.

[18] G. Feng, "Stability analysis of piecewise discrete-time linear systems," IEEE Transactions on Automatic Control, vol. 47, no. 7, pp. 1108$1112,2002$.

[19] M. Johansson and A. Rantzer, "Computation of piecewise quadratic Lyapunov functions for hybrid systems," IEEE Transactions on Automatic Control, vol. 43, no. 4, pp. 555-559, Apr 1998. 